\begin{tabular}{|c|c|}
\hline Title & Deflation Techniques for Computational Electromagnetism: Theoretical Considerations \\
\hline Author(s) & Igarashi, Hajime; W atanabe, Kota \\
\hline Citation & $\begin{array}{l}\text { IEEE T ransactions on Magnetics, 47(5), 1438-1441 } \\
\text { https://doi.org/10.1109/ MA G.2010.2094998 }\end{array}$ \\
\hline Issue Date & $2011-05$ \\
\hline Doc URL & http:/hdl.handle.net/2115/46102 \\
\hline Rights & $\begin{array}{l}\text { (c) } 2011 \text { IEEE. Personal use of this material is permitted. Permission from IEEE must be obtained for all other uses, in } \\
\text { any current or future media, including reprinting/republishing this material for advertising or promotional purposes, } \\
\text { creating new collective works, for resale or redistribution to servers or lists, or reuse of any copyrighted component of } \\
\text { this work in other works. }\end{array}$ \\
\hline Tyре & article (author version) \\
\hline File Information & IEEE_TM47_1438-1441.pdf \\
\hline
\end{tabular}

Instructions for use 


\title{
Deflation Techniques for Computational Electromagnetism: Theoretical Considerations
}

\author{
Hajime Igarashi, Member IEEE, Kota Watanabe, Member IEEE \\ Graduate School of Information Science and Technology, Hokkaido University, \\ Kita 14, Nishi 9, Kita-ku, 060-0814 Sapporo, Japan
}

\begin{abstract}
This paper describes deflation of finite element (FE) matrices for electromagnetic fields. The condition of the FE matrices is improved by the matrix deflation which replaces small eigenvalues with zeros. It is known that convergence of linear solvers for FE equations can be improved by using the AV method as well as explicit and implicit error correction (EC) methods which have been derived from the multigrid method. These numerical results are theorized on the basis of the matrix deflation. In particular, augmented matrices appeared in the AV and implicit EC methods are shown to have good conditioning after preconditioning. These results suggest that the above methods are based on a common mathematical principle.
\end{abstract}

Index Terms - matrix deflation, projection matrix, augmented matrix, finite element method, AV method

\section{INTRODUCTION}

$\mathbf{I}$ $\mathrm{T}$ is known that convergence of linear solvers used in finite element (FE) analysis of electromagnetic fields depends on matrix conditioning. Poor matrix conditioning often gives rise to severe problems in the FE analysis. For example, one has poor matrix conditioning when the elements are quite flat or distorted. Moreover, one encounters such a problem when the frequency or conductivity is set to small in the eddy current analysis based on the A method whose unknown variable is the vector potential.

The matrix deflation, which replaces small eigenvalues with zeros to improve matrix conditioning, would cure the above mentioned problems. The deflation technique has been applied to the conjugate gradient $(\mathrm{CG})$ method with application to boundary value problems [1], and also to diffusion problems for soil layers with significantly different permeability [2]. Moreover, the deflated CG method has been introduced on the basis of the Lanczos algorithm [3]. In computational electromagnetism, magnetostatic problems with large jumps in the magnetic permeability have been analyzed using the deflation method where the approximated eigenvectors corresponding to small eigenvalues are obtained from simplified FE models with local supports [4].

Besides the matrix deflation, the explicit error correction (EEC) and implicit error correction (IEC) techniques have been introduced to eliminate slowly converging components [5]. These methods can be recognized as a modified version of the multigrid method, and are also relevant to the AV method which employs an augmented FE matrix. Moreover, convergence of the CG method applied to FE analysis of magnetostatic problems with considerably flat elements has been shown to be drastically improved by the singularity decomposition (SD) [6], which has the same theoretical basis as the IEC. These two methods will be referred as the IEC-SD method hereafter.

This paper discusses the effectiveness of the matrix deflation

Manuscript received May 31, 2010. Corresponding author: H. Igarashi (email: igarashi@ssi.ist.hokudai.ac.jp). applied to FE analysis. The reason why the AV, IEC-SD, EEC give good conditioning will be explained from the theory of the matrix deflation. In particular, the mathematical property of the augmented matrices used in the AV and IEC-SD will be clarified. The numerical results will be shown to support the theoretical results.

\section{MATRIX DEFLATION}

\section{A. Projection matrix}

The matrix deflation is formulated on the basis of the projection matrices, which will be introduced below. Let us consider a system of linear equations

$$
A \boldsymbol{x}=\boldsymbol{b},
$$

where $A \in \mathbb{R}^{n \times n}$ is assumed to be symmetric positive definite. The solution vector $\boldsymbol{x}$ is decomposed into slowly and fast converging components as $\boldsymbol{x}=W \boldsymbol{y}+(\boldsymbol{x}-W \boldsymbol{y})$, where $W=\left[\boldsymbol{w}_{1}, \boldsymbol{w}_{2}, \ldots, \boldsymbol{w}_{k}\right] \in \mathbb{R}^{n \times k}$ and $\boldsymbol{w}_{i} \in \mathbb{R}^{n}, i=1,2, \ldots, k$ are linearly independent vectors. Here $k$ is set to sufficiently smaller than $n$. Two examples for choice of $W$ will be presented in sections III(c) and IV. Now A-orthogonality is imposed on the vectors $\boldsymbol{w}_{i}$ to $\boldsymbol{x}-W \boldsymbol{y}$ results in

$$
W^{t} A W \boldsymbol{y}=W^{t} A \boldsymbol{x} .
$$

Because $W^{t} A W \in \mathbb{R}^{k \times k}$ is regular, the slowly converging component $W \boldsymbol{y}$ can be expressed as

$$
\begin{aligned}
W \boldsymbol{y} & =W\left(W^{t} A W\right)^{-1} W^{t} A \boldsymbol{x} \\
& \equiv Q \boldsymbol{x},
\end{aligned}
$$

where $Q \in \mathbb{R}^{n \times n}$. Moreover, let us introduce the dual matrix $P=I-Q$ whose explicit form is given by

$$
P=I-W\left(W^{t} A W\right)^{-1} W^{t} A .
$$

Now the solution $\boldsymbol{x}$ can be expressed as $\boldsymbol{x}=Q \boldsymbol{x}+P \boldsymbol{x}$. It can be shown that $P$ is a projection matrix which satisfies $P^{2}=P$, and the same property holds for $Q$. Moreover, one can see that $P Q=0$ holds by definition, and find that $P$ and 
$A$ are commutable, namely, $A P=P^{t} A$, which leads to the above mentioned A-orthogonality

$$
(P \boldsymbol{x}, Q \boldsymbol{x})_{A}=\boldsymbol{x}^{t} P^{t} A Q \boldsymbol{x}=0 .
$$

\section{B. Effect of matrix deflation}

Let us assume that $W$ is composed of the orthonormal eigenvectors $\boldsymbol{w}_{i}, i=1,2, \ldots, k$ corresponding to the smallest $k$ eigenvalues $\lambda_{1} \leq \lambda_{2} \ldots \leq \lambda_{k}$ of $A$. In addition, we introduce $W^{*} \in \mathbb{R}^{n \times(n-k)}$ which consists of the remained eigenvectors corresponding to the eigenvalues $\lambda_{k+1} \leq \lambda_{k+2} \ldots \leq \lambda_{n}$.

In the deflation method, the slowly and fast converging components $Q \boldsymbol{x}, P \boldsymbol{x}$ are separately determined as follows: $Q \boldsymbol{x}$ is readily obtained from

$$
\begin{aligned}
Q \boldsymbol{x} & =W\left(W^{t} A W\right)^{-1} W^{t} A \boldsymbol{x} \\
& =W\left(W^{t} A W\right)^{-1} W^{t} \boldsymbol{b},
\end{aligned}
$$

where $W^{t} A W$ is easily inverted because $k \ll n$ as mentioned above. Note here that $W^{t} A W=\Lambda$ where $\Lambda=$ $\operatorname{diag}\left[\lambda_{1}, \ldots, \lambda_{k}\right]$. On the other hand, the component $P \boldsymbol{x}$ can be determined by solving

$$
A P \boldsymbol{x}=P^{t} A \boldsymbol{x}=P^{t} \boldsymbol{b} .
$$

One can see that the symmetric matrix $P^{t} A$ has $k$ zero eigenvalues because $P^{t} A W=A W-A W\left(W^{t} A W\right)^{-1} W^{t} A W=$ $A W-A W=0$ holds. Moreover, it follows from $P^{t} A W^{*}=$ $A W^{*}-A W\left(W^{t} A W\right)^{-1} W^{t} A W^{*}=A W^{*}=W^{*} \Lambda^{*}$, where $\Lambda^{*}=\operatorname{diag}\left[\lambda_{k+1}, \ldots, \lambda_{n}\right]$, that $P^{t} A$ has eigenvalues $\lambda_{k+1}, \ldots, \lambda_{n}$. It is, therefore, concluded that the small eigenvalues of $A$ are replaced with zeros by the matrix deflation while the other eigenvalues are remained unchanged. This means that the condition number $\operatorname{cond}(A)=\lambda_{n} / \lambda_{1}$ is decreased to $\operatorname{cond}\left(P^{t} A\right)=\lambda_{n} / \lambda_{k+1}$ after the deflation. Note here that $P^{t} A$ in (7) is singular. There exist, however, solutions to (7) because $P^{t} \boldsymbol{b}$ is perpendicular to $W$ which expresses the kernel of $P^{t} A$. Although $\boldsymbol{x}$ is arbitrary to an extent of addition of $W, P \boldsymbol{x}$ is uniquely determined because of $P W=0$.

\section{AnAlysis of Related Methods}

This section discusses the properties of the EEC, IEC-SD as well as AV methods through the theory of the matrix deflation.

\section{A. EEC method}

Let us consider the simplified multigrid algorithm with double layered V-cycle applied to (1), which has the following steps: (a) solution of (1) and evaluation of residual $\boldsymbol{r}=\boldsymbol{b}-A \boldsymbol{x}^{\prime}$, (b) restriction and solution of the restricted equation $\Pi^{t} A \Pi z=\Pi^{t} \boldsymbol{r}$, (c) prolongation and error correction $\boldsymbol{x}=\boldsymbol{x}+\Pi \boldsymbol{z}$, where $\Pi$ and $\Pi^{t}$ are prolongation and restriction matrices. Inspired from the multigrid method, Iwashita et al. introduced EEC method [5], which uses the matrix $W$, in stead of $\Pi$, to decompose $\boldsymbol{x}$ into $\boldsymbol{x}=\boldsymbol{x}^{\prime}+W \boldsymbol{z}$. Then (1) is rewritten in the form

$$
A \boldsymbol{x}^{\prime}=\boldsymbol{b}-A W \boldsymbol{z} .
$$

Moreover, the Galerkin condition that the residual $\boldsymbol{b}-A \boldsymbol{x}^{\prime}+$ $A W \boldsymbol{z}$ is perpendicular to $W$ imposed on (8) yields the equation for $\boldsymbol{z}$ as

$$
W^{t} A W \boldsymbol{z}=W^{t}\left(\boldsymbol{b}-A \boldsymbol{x}^{\prime}\right) .
$$

At the first stage of the EEC, (8) is solved for $\boldsymbol{x}^{\prime}$ by setting $\boldsymbol{z}=0$. Next, inserting $\boldsymbol{x}^{\prime}$ into the right-hand side of (9), which is solved for $\boldsymbol{z}$. Then $\boldsymbol{z}$ is substituted into the right-hand side of (8). These iterative processes continue until convergence. The EEC is expected to improve the convergence in solution of (1) because it has the same algorithm as the multigrid method. However, there have not been mathematical discussions on the property of the EEC, which will be given in the following.

To consider the property of (8) and (9), $\boldsymbol{z}$ is eliminated from them to obtain

$$
\begin{gathered}
\left\{I-A W\left(W^{t} A W\right)^{-1} W^{t}\right\} A \boldsymbol{x}^{\prime}= \\
\left\{I-A W\left(W^{t} A W\right)^{-1} W^{t}\right\} \boldsymbol{b} .
\end{gathered}
$$

One can find that (10) is identical with the deflated equation (7) because $P \boldsymbol{x}^{\prime}$ is identical with $P \boldsymbol{x}$. Therefore, assuming that $W$ is composed of the eigenvectors corresponding to the smallest eigenvalues of $A$, it is concluded that the EEC improves the matrix conditioning by replacing the small eigenvalues with zeros in the same manner as the matrix deflation.

\section{B. IEC-SD method}

In the IEC-SD method, (8) and (9) are coupled in a form

$$
\left[\begin{array}{cc}
A & A W \\
W^{t} A & W^{t} A W
\end{array}\right]\left[\begin{array}{c}
\boldsymbol{x}^{\prime} \\
\boldsymbol{z}
\end{array}\right]=\left[\begin{array}{c}
\boldsymbol{b} \\
W^{t} \boldsymbol{b}
\end{array}\right],
$$

which is solved for $\boldsymbol{x}^{\prime}$ and $\boldsymbol{z}$. Note here that (11) can also be derived directly from the deflated equation (7) and (9). The augmented matrix in (11), denoted by $B$ hereafter, has been used for preconditioning of ill-conditioned system of linear equations [7].

Discussion based on a toy problem and numerical examples in [6] suggest that preconditioning of $B$ yields wellconditioned systems in contrast to $A$. A mathematical discussion on this property will be given in the followings.

The eigenvalues of $B$ are obtained from

$$
\begin{gathered}
B\left[\begin{array}{c}
W \\
-I_{k}
\end{array}\right]=\left[O_{n+k}\right], \quad B\left[\begin{array}{c}
W \\
I_{k}
\end{array}\right]=2\left[\begin{array}{c}
W \\
I_{k}
\end{array}\right] \Lambda, \\
B\left[\begin{array}{c}
W^{*} \\
O_{k \times(n-k)}
\end{array}\right]=\left[\begin{array}{c}
W^{*} \\
O_{k \times(n-k)}
\end{array}\right] \Lambda^{*},
\end{gathered}
$$

where $I$ and $O$ represent unit and zero matrices. It can be concluded from (13) that the eigenvalues are $k$ zeros, $2 \lambda_{1}, \ldots, 2 \lambda_{k}$ $\lambda_{k+1}, \ldots, \lambda_{n}$ [11], where $\lambda_{i}, i=1,2, \ldots, n$ are the eigenvalues of $A$. It follows from this result that $\operatorname{cond}(B)=\lambda_{n} /\left(2 \lambda_{1}\right)$, thus, the conditioning of $B$ is still poor if $\operatorname{cond}(A)$ is large. This suggests that preconditioning plays a crucial role in improvement of conditioning of $B$.

To show this theoretically, we first consider the asymptotic property of the preconditioned matrix of $B$, denoted by $\tilde{B}$. We introduce here a parameter $p$ so that $\operatorname{cond}(A)$ becomes large when $p \rightarrow 0$. For instance, $p$ would be thickness of thin finite elements, or $\omega$ in the A method which 
will be discussed later. It is assumed that $\lambda_{i} \rightarrow 0, i=$ $1,2, \ldots, k$ as $p \rightarrow 0$ while $\lambda_{i}>0, i=k+1, \ldots, n$. Hence $A W \rightarrow O$ in this limit. For simplicity, the diagonal scaling is assumed for the preconditioner consisting of diagonal matrices $D^{-1 / 2}=\operatorname{diag}\left[1 / \sqrt{a_{11}}, \ldots, 1 / \sqrt{a_{n n}}\right]$ and $\Lambda^{-1 / 2}=\operatorname{diag}\left[1 / \sqrt{\lambda_{1}}, \ldots, 1 / \sqrt{\lambda_{k}}\right]$, where $a_{i i}, i=1,2, \ldots, n$ are the diagonal components of $A$. Then one finds that the preconditioned matrix

$$
\tilde{B}=\left[\begin{array}{cc}
\tilde{A} & D^{-1 / 2} A W \Lambda^{-1 / 2} \\
\Lambda^{-1 / 2} W^{t} A D^{-1 / 2} & I_{k}
\end{array}\right],
$$

where $\tilde{A}=D^{-1 / 2} A D^{-1 / 2}$ which is the preconditioned matrix of $A$ obtained by the diagonal scaling, has the limit

$$
\tilde{B} \rightarrow\left[\begin{array}{cc}
\tilde{A} & O_{n \times k} \\
O_{k \times n} & I_{k}
\end{array}\right]
$$

as $p \rightarrow 0$. The eigenvalues of $\tilde{A}$ will be written as $\tilde{\lambda}_{i}, i=$ $1,2, \ldots, n$, and the same rule is adopted to the other quantities relevant to $\tilde{A}$. Thus, in this limit, the eigenvalues of $\tilde{B}$ are $k$ zeros, $k$ ones and positive values $\tilde{\lambda}_{k+1} \leq \ldots \leq \tilde{\lambda}_{n}$. When $p$ slightly increases from zero, the eigenvalues of $\tilde{B}$ would stay near the above values because of eigenvalue continuity. However, one can see that

$$
\tilde{B}\left[\begin{array}{c}
D^{1 / 2} W \\
-\Lambda^{1 / 2}
\end{array}\right]=\left[O_{n+k}\right] .
$$

It follows from (16) that the zero eigenvalues remain unchanged even if $p$ increases. It is, therefore, concluded that $\tilde{B}$ has no small eigenvalues which deteriorate the convergence in contrast to $\tilde{A}$.

The eigenvalues of $\tilde{B}$ can be found when assuming that $A$ in (1) is already preconditioned by the diagonal scaling, namely, $A=\tilde{A}$. Equation (1) would be still ill-conditioned after preconditioning when $p$ is small. Then one can derive the following theorem.

Theorem 1 The eigenvalues of $\tilde{B}$ generated from $\tilde{A}$ and $\tilde{W}$, which consists of the eigenvectors corresponding to the smallest $k$ eigenvalues of $\tilde{A}$, are $k$ zeros and $1+\tilde{\lambda}_{1}, 1+\tilde{\lambda}_{2}, \ldots, 1+\tilde{\lambda}_{k}$, $\tilde{\lambda}_{k+1}, \ldots, \tilde{\lambda}_{n}$.

proof: Because $A=\tilde{A}$, one can set $D=I_{k}$ in (14). Now the eigenvalues of $\tilde{B}$ can be found from

$$
\begin{gathered}
\tilde{B}\left[\begin{array}{c}
\tilde{W} \\
-\tilde{\Lambda}^{1 / 2}
\end{array}\right]=\left[O_{n+k}\right], \\
\tilde{B}\left[\begin{array}{c}
\tilde{W}^{*} \\
O_{k}
\end{array}\right]=\left[\begin{array}{c}
\tilde{W}^{*} \\
O_{k}
\end{array}\right] \tilde{\Lambda}^{*}, \\
\tilde{B}\left[\begin{array}{c}
\tilde{W} \\
\tilde{\Lambda}^{-1 / 2}
\end{array}\right]=\left[\begin{array}{c}
\tilde{W}^{-1 / 2} \\
\tilde{\Lambda}^{-1 / 2}
\end{array}\right]\left(I_{k}+\tilde{\Lambda}\right),
\end{gathered}
$$

where $\tilde{W}^{*}$ contains the eigenvectors of $\tilde{A}$ corresponding to its eigenvalues $\tilde{\lambda}_{k+1} \leq \ldots \leq \tilde{\lambda}_{n}$. Hence it is clear that the eigenvalues of $\tilde{B}$ are $\bar{k}$ zeros and $1+\tilde{\lambda}_{1}, 1+\tilde{\lambda}_{2}, \ldots, 1+\tilde{\lambda}_{k}$, $\tilde{\lambda}_{k+1}, \ldots, \tilde{\lambda}_{n} \cdot \diamond$

It can be concluded from theorem 1 that the conditioning of $B$ is effectively improved after the diagonal scaling especially when $\lambda_{1} \ll 1$.

\section{AV method for eddy current problems}

Here we focus on the FE formulations for eddy current problems, to which the concept of the matrix deflation will be applied. The domain is assumed to be divided into finite elements which have $f$ faces, $e$ edges and $n$ nodes. The A method solves the FE equation of the form [13]

$$
\left(C^{t} N C+\mathrm{j} \omega S\right) \boldsymbol{a}=\boldsymbol{j},
$$

where $C \in \mathbb{R}^{f \times e}$ is the discrete counterpart of curl operator [12]. The entities of $N \in \mathbb{R}^{f \times f}$ and $S \in \mathbb{R}^{e \times e}$ are given by $\left(\nu M_{i}, M_{j}\right)$ and $\left(\sigma \boldsymbol{N}_{i}, \boldsymbol{N}_{j}\right)$ where $\boldsymbol{M}$ and $\boldsymbol{N}$ are the basis functions of facet and edge elements. It is known that $\operatorname{rank}\left(C^{t} N C\right)=e-n+1$, thus $C^{t} N C$ is singular. For simplicity, $\sigma$ is assumed to be positive so that $S$ is regular. In this case, the FE matrix in (20), denoted by $K$, becomes nearly singular when $\omega$ becomes small. This is the reason why the A method has slow convergence.

To overcome this difficulty, we adopt the matrix deflation to $K$. We can employ $G \in \mathbb{R}^{e \times n}$, the discrete counterpart of grad operator which satisfies $C G=O$, for $W$ since $K G \rightarrow O$ as $\omega$ approaches zero which corresponds to $p$ in the above discussion. The resultant deflated equation takes the same form as (7) where $P$ is now defined by

$$
P=I-G\left(G^{t} S G\right)^{-1} G^{t} S .
$$

In (21), the matrix inversion is defined in a sense of pseudoinverse if $G^{t} S G$ is singular. As in the derivation of (11), the linear equations with the augmented matrix can be derived from (7) by introducing an additional variable $\boldsymbol{v}$ which satisfies $G^{t} S G \boldsymbol{v}=-G^{t} S \boldsymbol{a}+G^{t} \boldsymbol{j} /(\mathrm{j} \omega)$ as

$$
\left[\begin{array}{cc}
K & \mathrm{j} \omega S G \\
\mathrm{j} \omega G^{t} S & \mathrm{j} \omega G^{t} S G
\end{array}\right]\left[\begin{array}{l}
\boldsymbol{a} \\
\boldsymbol{v}
\end{array}\right]=\left[\begin{array}{c}
\boldsymbol{j} \\
G^{t} \boldsymbol{j}
\end{array}\right],
$$

which is nothing but the equation of the AV method, where the charge conservation law $G^{t} \boldsymbol{j}=0$ is enforced. Since the augmented matrix in (22) has the same structure as $\tilde{B}$ in (11), it would have good conditioning after preconditioning. Indeed, this property has been shown experimentally in [13] and theoretically in [14].

In summary, it is concluded that the EEC, IEC-SD and AV methods are based on the common mathematical principle as the matrix deflation.

\section{NUMERICAL RESULTS}

We consider a simple magnetostatic problem in which a thin magnetic layer is immersed in uniform external magnetic field as shown in Fig.1. The domain is uniformly discretized into edge-based hexahedral FEs. Note that the FEs become flat as $d$ decreases so that the matrix conditioning becomes poor.

The unknown $\boldsymbol{x}$ is composed of the line integrals of the vector potential along the $\mathrm{FE}$ edges. The magnetostatic FE equation, $K_{0} \boldsymbol{x}=\boldsymbol{j}, K_{0}=C^{t} N C$ is solved using the deflation method which treats with the deflated equation of the form (7). The deflated CG method [3] is employed for computer implementation. This problem is also analyzed using the conventional ICCG method without special treatments and the IEC-SD method, the latter of which solves the augmented 
FE equation of the form (11). The deflation matrix $W$ is constructed as follows [6]: the FE edges whose direction is parallel to $x$ or $y$ axis are classified into $m$ groups where the elements in a group have the same $(x, y)$ coordinates. For each group, a vector $\boldsymbol{w}_{i}$ is defined so that the entities corresponding to the edges in the group are set to $a$, a constant, and others are set to zero. This configuration gives small magnetic flux because the magnetic circulation along edges on an element face is small. The magnetic energy, thus, obeys $\boldsymbol{w}_{i}^{t} K_{0} \boldsymbol{w}_{i} / 2 \rightarrow 0$ as $d \rightarrow 0$. This means that $\boldsymbol{w}_{i}$ stands for the eigenvector corresponding to the eigenvalue of $K_{0}$ which approaches zero as $d \rightarrow 0$. In this way, the vectors $\boldsymbol{w}_{i}, i=1,2, \ldots, m$ is generated to construct $W$. In the numerical computation, $a$ is set to $1 \times 10^{-3}$.

The resultant solution times depending on the aspect ratio $1 / d$ are shown in Fig.2. One finds that convergence of the deflation and IEC-SD methods is almost independent of $1 / d$ in contrast to that of the ICCG. Figure 3 shows the eigenvalues of the matrices $\tilde{A}$ and $\tilde{B}$ preconditioned with the diagonal scaling. The small eigenvalues, attributed to flatness of the elements, are eliminated in $\tilde{B}$ as expected.

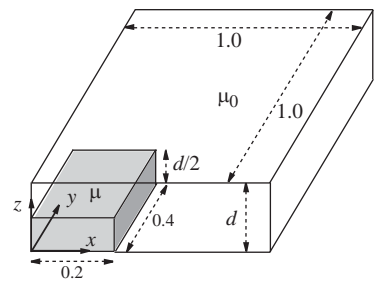

Fig.1 Magnetostatic problem, where uniform magnetic induction $B_{z}$ is imposed to a magnetic material, $\mu=1 \times 10^{4} \mu_{0}$

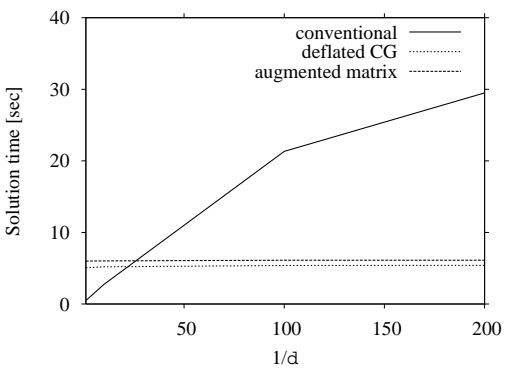

Fig.2 Solution times

V. Conclunsions

In this paper, the matrix deflation has been discussed especially focusing on application to analysis of electromagnetic fields. In this method, small eigenvalues of a matrix are replaced with zeros for improvement of matrix conditioning. The reason why the EEC, IEC-SD as well as AV methods can improve matrix conditioning has been clarified through the theory of the matrix deflation. In particular, the mathematical properties of the augmented matrix used in the IEC-SD and AV methods have been discussed in detail. Numerical results have shown that both deflated CG method and IEC-SD keep good convergence even if the aspect ratio of finite elements increases in contrast to the conventional ICCG.

In practical FE modeling, flat FEs often present in, for example, thin magnetic-shielding region and skin layers in eddy current problems. The present method would be effective to reduce computational times for these problems.

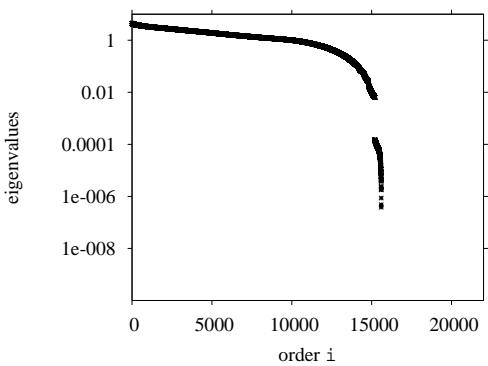

(a) original matrix $\tilde{A}$

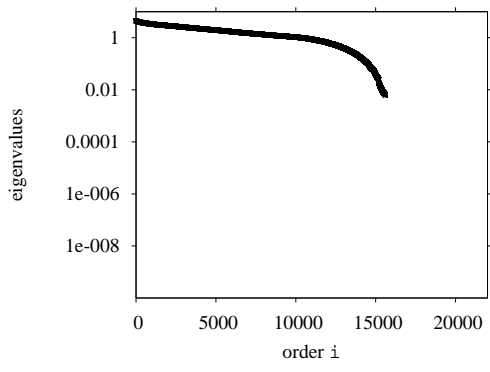

(b) augmented matrix $\tilde{B}$

Fig.3 Eigenvalues $\tilde{\lambda_{i}}$ of original and augmented FE matrices which are preconditioned with diagonal scaling.

\section{REFERENCES}

[1] R.A. Nicolaides, "Deflation of Conjugate Gradients with application to boundary value problems," SIAM J. Numer. Anal., vol.24, pp.355-365, 1987.

[2] C. Vuik, A. Segal, J.A. Meijerink, "An efficient preconditioned CG method for the solution of a class of layered problems with extreme contrasts in the coefficients," J. Comp. Phys., vol.152, pp.385-403, 1999.

[3] Y. Saad, M. Yeung, J. Erhel, F. Guyomarch, "A deflated version of the conjugate gradient algorithm," SIAM J. Sci. Comput., vol.21, no.5, pp.1909-1926, 2000.

[4] H. De Gersem, K. Hameyer,"A deflated iterative solver for magnetostatic finite element models with large differences in permeability," Eur. Phys. J. AP., vol.13, no.1, pp.45-49, 2001.

[5] T. Iwashita, T. Mifune, M. Shimasaki, "Similarities between implicit correction multigrid method and A-phi formulation in electromagnetic field analysis," IEEE Trans. Magn., vol.44, no.6, pp.946-949, 2008.

[6] A. Kameari, "Improvement of ICCG convergence for thin elements in magnetic field analyses using the finite-element method," IEEE Trans. Magn., vol.44, no.6, pp.1178-1181, 2008.

[7] A. Padiy, O. Axelsson, B. Polman, "Generalized Augmented Matrix Preconditioning Approach and its Application to Iterative Solution of Ill-Conditioned Algebraic Systems," SIAM J. Matrix Anal. Appl., vol.22, no.3, pp.793-818, 2000.

[8] C. Vuik, A. Segal, J.A. Meijerink, G.T. Wijma, "The construction of projection vectors for a deflated ICCG method applied to problems with extreme contrasts in the coefficients," J. Comp. Phys., vol.172, pp.426450, 2001.

[9] L. Mansfield, "Damped Jacobi preconditioning and coarse grid deflation for conjugate gradient iteration on parallel computers," SIAM J. Sci. Statist. Comput., vol.12, pp.1314-1323, 1991.

[10] J. Frank, C. Vuik, " On the construction of deflation-based preconditioners," SIAM J. Sci. Statist. Comput., vol.23, pp.442-462, 2001.

[11] K. Mifune, S. Moriguchi, T. Iwashita, M. Shimasaki,“ A basic study on the implicit error correction method and explicit error correction method for the finite element method using a mash with high aspect ratio," Technical paper of IEE, SA-08-7, 2008 (in Japanese).

[12] A. Bossavit, "Computational electromagnetism", Academic press, 1998

[13] H. Igarashi and T. Honma, "On the convergence of ICCG applied to finite element equation for quasi-static fields", IEEE Trans. Magn., vol. 38, no.2, pp.565-568, 2002.

[14] H. Igarashi, N.Yamamoto, "Effect of preconditioning in edge-based finite-element method", IEEE Trans. Magn., vol.44, no.6 pp.942-945, 2008. 